\title{
Síndrome de Stiff-Person associada à poliendocrinopatia autoimune: um relato de caso
}

\author{
Stiff-Person syndrome associated with autoimmune polyiendocrinopathy: a case report \\ Síndrome de Stiff-Person asociado a la poliendocrinopatía autoinmune: informe de caso
}

Laíza Emrich Crunivel ${ }^{1 *}$, Cibele da Silveira Corrêa ${ }^{1}$, Daiane Cristine Silva Lopes ${ }^{2}$, Fílippe da Cruz Machado Teixeira' ${ }^{1}$.

\section{RESUMO}

Objetivo: Demonstrar uma associação de síndrome da pessoa-rígida (SPR) com diabetes mellitus tipo 1 e tireoidite de Hashimoto em uma paciente do sexo feminino diagnosticada aos 35 anos, que apresentou boa resposta terapêutica ao uso de benzodiazepínico e melhora significativa da sua qualidade de vida. Detalhamento do caso: A síndrome de stiff-person (SPS) ou é caracterizada por uma intensa rigidez axial e proximal de membros associados a espasmos. Há um componente autoimune marcante da doença caracterizado pela presença do anticorpo anti-GAD. O presente caso clínico demonstra uma associação de SPR com diabetes mellitus tipo 1 e tireoidite de Hashimoto em uma paciente do sexo feminino diagnosticada aos 35 anos, que apresentou boa resposta terapêutica ao uso de benzodiazepínico e melhora significativa da sua qualidade de vida. Apesar de se tratar de uma condição rara, é uma condição que merece ser investigada em pacientes com acometimento muscular e poliendocrinopatias para realização de diagnóstico e tratamentos adequados. Considerações finais: Apesar de se tratar de uma condição rara, é uma condição que merece ser investigada em pacientes com acometimento muscular e poliendocrinopatias para realização de diagnóstico e tratamentos adequados.

Palavras-chave: Doenças raras, Neurologia, Poliendocrinopatias.

\section{ABSTRACT}

Objective: This present clinical case demonstrates an association between the stiff person syndrome (SPS) and diabetes mellitus 1 and Hashimoto hypothyroidism in a female pacient diagnosed when 35 years that presented an amazing response to benzodiazepine and significant improvement in her life's quality. Case details: Stiff person syndrome is characterized by a huge axial and proximal members stiffness associated with spasms. There is a memorable immune component of this disease characterized by antibody anti-GAD. This present clinical case demonstrates an association between this syndrome and diabetes mellitus 1 and Hashimoto hypothyroidism in a female pacient diagnosed when 35 years that presented an amazing response to benzodiazepine and significant improvement in her life's quality. Despite the fact that the disease is rare, it is a condition that deserves to be investigated in pacients who complain about muscles disease and polyiendocrinopathies to achieve correct diagnosis and treatment. Final considerations: Although it is a rare condition, it is a condition that deserves to be investigated in patients with muscle involvement and polyiendocrinopathies for diagnosis and appropriate treatments.

Key words: Rare diseases, Neurology, Polyiendocrinopathies.

\section{RESUMEN}

Objetivo: El presente caso clínico demuestra una asociación de síndrome de persona-rígida (SPR) con diabetes mellitus tipo 1 y tiroiditis de Hashimoto en una paciente de sexo femenino diagnosticada a los 35 años, que presentó una buena respuesta terapéutica al uso de benzodiazepínico y mejoró significativamente su calidad de vida. Details del case: El síndrome de stiff-person (SPS) es caracterizada por una intensa rigidez axial y proximal de miembros asociados con espasmos. Existe un componente autoinmune notable de la enfermedad que se caracteriza por la presencia de anticuerpos anti-GAD. El presente caso clínico demuestra una asociación de SPR con diabetes mellitus tipo 1 y tiroiditis de Hashimoto en una paciente de

1 Universidade de Uberaba (UNIUBE), Uberaba - MG. *E-mail: laizaemrich@yahoo.com.br

2 Instituto Master de Ensino Presidente Antônio Carlos (IMEPAC), Araguari - MG. 
sexo femenino diagnosticada a los 35 años, que presentó una buena respuesta terapéutica al uso de benzodiazepínico y mejoró significativamente su calidad de vida. Aunque sea una condición rara, merece una investigación en pacientes con acometimiento muscular y poliendocrinopatías para realización de diagnóstico y tratamientos adecuados. Consideraciones finales: Aunque sea una condición rara, merece una investigación en pacientes con acometimiento muscular y poliendocrinopatías para realización de diagnóstico y tratamientos adecuados.

Palabras clave: Enfermedades raras, Neurología, Poliendocrinopatías.

\section{INTRODUÇÃO}

O termo "homem rígido" foi introduzido pela primeira vez pelos médicos Moersch e Woltman em 1956 para descrever as características clínicas da "rigidez muscular flutuante e progressiva" e espasmos observados em 14 pacientes atendidos na Clínica Mayo por um período de 32 anos. A síndrome de stiff-person ou síndrome da pessoa-rígida (SPR) é uma rara afecção neurológica caracterizada por uma intensa rigidez axial e proximal de membros, com espasmos inesperados, devido a uma atividade contínua das unidades motoras que são progressivos e podem ser fatais. Existem diversas variantes da SPR, incluindo Síndrome de tronco rígido, síndrome de membro rígido, encefalomielite progressiva com rigidez, SPR-plus e SPR paraneoplásica (MOERSCH FP e WOLTMAN HW, 1956).

A prevalência dessa patologia é estimada em 1/1.000.000, sem predileção por etiologia e com uma relação de 2:1 de mulheres para homens, sendo a faixa etária mais acometida de 35 a 50 anos (APODACA, et al., 2018). Frequentemente, outras doenças autoimunes estão associadas, como diabetes mellitus tipo 1 em até $64 \%$ das vezes, $28,6 \%$ dos pacientes apresentam alguma tireoidopatia autoimune, patologias psiquiátricas podem estar associadas em até $78 \%$ dos casos, tumores endócrinos são associações mais raras, presentes em 7\% dos casos (LINO VC, 2016).

A associação de autoanticorpos contra a descarboxílase do ácido glutâmico (anti-GAD) são encontrados em cerca de 60 a $80 \%$ dos casos SPR. A descarboxílase do ácido glutâmico (GAD) é uma enzima citoplasmática que acelera a conversão de ácido glutâmico a ácido gama-aminobutírico (GABA), um neurotransmissor inibitório presente no cérebro e medula espinhal. Ela é sintetizada principalmente em neurônios gabaérgicos pré-sinápticos no sistema nervoso central e nas células beta nas ilhotas de Langerhans no pâncreas (SOLIMENA M, et al., 1990).

As manifestações clínicas mais comuns são redução da mímica facial, espasmos laríngeos, desordens cognitivas, aumento do reflexo patelar, rigidez muscular, espasmos sobrepostos pela rigidez adjacente, contração conjunta da musculatura agonista e antagonista, hiperlordose, marcha prejudicada, dor e quedas. O diagnóstico pode ser feito através da associação clínica e eletroneuromiografia caracterizada por uma atividade contínua da unidade motora tipicamente diminuída ou interrompida por benzodiazepínicos ou anestésicos (HELFGOTT SM, 1999).

O tratamento permite boa qualidade de vida e nele são utilizados benzodiazepínicos e baclofen como as principais intervenções terapêuticas, abordando a rigidez muscular e os espasmos nestes casos. Entretanto, há estudos que descrevem melhora acentuada da rigidez muscular e dos espasmos após uso de novas drogas anticonvulsivante (levetiracetam) em alguns pacientes (CARRILHO P, et al., 2014).

O presente estudo teve como objetivo relatar um caso de uma patologia rara, denominada Síndrome de Stiff-Person associada à poliendocrinopatias em uma paciente do sexo feminino diagnosticada aos 35 anos de idade, que teve boa resposta ao uso de benzodiazepinícos no seu tratamento, atendida em um hospital universitário em Minas Gerais.

\section{DETALHAMENTO DE CASO}

Paciente do sexo feminino, parda, 46 anos, relata que em fevereiro de 2008 iniciou quadro de enrijecimento lombar, principalmente do lado esquerdo, associado à hiperlordose, marcha espástica e posteriormente a quedas múltiplas sempre durante movimentos de descida. Relata, ainda, que na época sentia espasmos 
involuntários em membros, principalmente em período noturno. Simultaneamente ao quadro, apresentou diminuição aguda da acuidade visual, logo foi feito o diagnóstico de Diabetes Mellitus tipo 1 e iniciou-se o tratamento com insulinoterapia.

As quedas continuaram e cada vez se tornaram mais frequentes, o que a levou a passar por diversas especialidades como ortopedia e reumatologia, mas sem nenhum diagnóstico. Iniciou-se então psicoterapia e acompanhamento psiquiátrico, acreditando se tratar de um quadro psicossomático, entretanto, mesmo em tratamento o seu quadro se agravava e as quedas tornavam-se ainda mais frequentes. Nessa época, a paciente começou a evitar andar, passando maior parte do tempo acamada por medo de novas quedas, e sempre que precisava se locomover só o fazia com apoio de bengala.

Em 2016 procurou um neurologista, o qual suspeitou do diagnóstico da Síndrome de Stiff-Person e solicitou exame autoanticorpos antidescarboxilase do ácido glutâmico (anti-GAD) com valor $158 \mathrm{Ul} / \mathrm{mL}$ (Valor de referência: Inferior a $10 \mathrm{Ul} / \mathrm{ml}$ ), eletroneuromiografia sem alterações, com exceção do nervo extensor digitorum brevis bilateralmente com discreto sinal de desoneração crônica, eletroencefalograma e tomografia não contrastada de crânio sem alterações.

Apesar de exames complementares não sugestivos, devido a forte suspeição clínica foi prescrito o benzodiazepínicos Alprazolam $1 \mathrm{mg}$ de $8 \mathrm{em} 8$ horas para o tratamento da mesma e após boa resposta com o tratamento proposto foi possível fechar o diagnóstico de SPS. Posteriormente, foi realizado a troca da medicação por Clonazepam $2 \mathrm{mg}$ de $8 \mathrm{em} 8$ horas, o qual apresentou melhor resposta terapêutica com remissão quase completa do quadro.

No final de 2019, realizando exames de rotina, apresentou hormônio estimulante da tireoide TSH com valor de $6 \mathrm{mU} / \mathrm{L}$ (Valor de referência: 0,3 a 4,0 Um/L), T4 livre 0,06 ng/dl (Valor de referência: 0,9 a 1,8ng/dl) e anticorpos anti- tireoperoxidase (anti-TPO) $1891 \mathrm{UI} / \mathrm{ml}$ (Valor de referência: Inferior a $15 \mathrm{UI} / \mathrm{ml}$ ), foi feito então o diagnóstico de tireoidite de Hashimoto e prescrito Levotiroxina $50 \mathrm{mcg}$ para a mesma.

Atualmente, durante períodos estressantes, ainda apresenta espasmos involuntários em membros inferiores, mas a frequência de quedas se limitou a três vezes no último ano, referindo grande melhora de sua qualidade de vida com o uso adequado de terapia medicamentosa proposta. Está em uso de Levotiroxina $50 \mathrm{mcg} 1$ comprimido pela manhã, Clonazepam 2mg de 8 em 8 horas, Insulina NPH 28 UI pela manhã e 10 $\mathrm{UI}$ a noite. $\mathrm{E}$ os exames mais atuais demonstraram bom controle das endocrinopatias, com TSH 2,06 mU/L, T4 livre 1,3n g/dl, glicemia em jejum $91 \mathrm{mg} / \mathrm{dl}$ (Valor de referência: 60 a 99mg/dl) e hemoglobina glicada igual a $6,5 \%$ (Valor de referência: 4 a 5,4\%). Devido a estabilidade do quadro clínico, paciente realiza acompanhamento anual com o Neurologista.

\section{DISCUSSÃO}

A síndrome do "homem rígido" foi descrita pela primeira vez em 1956 por Moersch e Woltman. Juntamente com observações de 13 outros casos, eles descreveram um homem de 49 anos com rigidez progressiva no pescoço, ombros e parte superior das costas, espasmos musculares dolorosos episódicos e dificuldade para caminhar. Desde então, várias descrições de casos foram feitas. O termo "homem rígido" foi substituído recentemente pela "síndrome da pessoa rígida" (SPS), de gênero neutro, que ganhou força significativa depois que Blum P e Jankovic J (1992); relataram que aproximadamente 20 dos 84 casos relatados entre 1967 e 1991 eram do sexo feminino.

A SPS é uma afecção incomum que se mostra com espasmos musculares associados a rigidez e contraturas dolorosas, sobretudo de musculatura axial. Frequentemente associa-se a diabetes do tipo 1 e, mais incomumente a outras doenças autoimunes, como vitiligo e tireoidite, o que mostra mecanismos patológicos compartilhados em tais doenças. Na SPR encontram-se elevados níveis de anticorpo anti-GAD em $60 \%$ dos pacientes, indicador considerado como padrão ouro para o diagnóstico da síndrome. A GAD é uma enzima que catalisa a descarboxilização do ácido gama-aminobutírico (GABA), o qual é um neurotransmissor inibitório no sistema nervoso central. A deterioração das vias GABAérgicas, devido à diminuição do GABA no cérebro, leva à atividade excitatória excessiva dos neurônios motores da medula, resultando em rigidez e espasmos que identificam a pessoa com essa patologia (LIRAN O, et al., 2015). 
A SPR é frequentemente associada com outras doenças autoimunes; de acordo com Espay AJ e Chen R (2006), mais de $80 \%$ dos pacientes terão pelo menos outra endocrinopatia associada. Anemia perniciosa, tireoidopatia autoimune e Diabetes Mellitus tipo 1 (DM1) estão presentes em menos de 5\%, 10-25\% e 35$60 \%$, respectivamente, em associação com essa síndrome. Ao diagnosticar um paciente com SPR, os médicos devem sempre suspeitar quanto à coexistência de doenças autoimunes não diagnosticadas associadas e realizar exames para rastreio das mesmas.

A paciente em questão foi diagnosticada aos 31 anos com DM1, normalmente a idade média para diagnóstico dessa patologia autoimune isoladamente ocorre entre 5 e 19 anos de idade. Já a idade média para o diagnóstico da Síndrome de Stiff-Person ocorre entre a terceira e quinta década de vida de acordo com Apodaca FJC, et al. (2018), o que vai de encontro à paciente em questão, diagnosticada aos 39 anos. Além da associação com DM1, essa paciente apresentou ainda uma tireoidite de Hashimoto, a qual aparece em menor prevalência em pacientes SPR, mas que reafirma a apresentação com poliendocrinopatias associadas.

A paciente relatada no presente artigo tinha altos títulos anti-GAD no momento do diagnóstico da SPR. Ainda não está claro se os anticorpos anti-GAD são diretamente patogênicos in vivo, ao contrário do diabetes e existem diferentes explicações possíveis para isso. Enquanto os títulos séricos de anticorpos anti-GAD no SPS são altos o suficiente para produzir danos endócrinos, os diabéticos têm títulos séricos mais baixos que provavelmente são insuficientes para atravessar a barreira hematoencefálica e levar a danos no sistema nervoso central (SNC).

Títulos extremamente altos de anticorpos anti-GAD no SPS podem desencadear autoimunidade de antígenos múltiplos e o desenvolvimento de outras doenças autoimunes concomitantes, como patologias da tireoide, mas não necessariamente vice-versa (GRIMALDI LM, et al., 2013). Historicamente, títulos anti-GAD não demonstraram correlação com gravidade ou duração da doença, no entanto, estudos recentes como o de Rakocevic G, et al. (2004), relataram que pacientes com SPR com piora da incapacidade no seguimento têm títulos anti-GAD mais altos, o que não ocorreu com essa paciente após o início do tratamento.

A fisioterapia para reduzir o movimento espasmódico é útil, mas algumas terapias também podem aumentar o tônus muscular e espasmos. A terapia cognitivo-comportamental pode ajudar a reduzir a rigidez relacionada à ansiedade relatada em um estudo de caso realizado por Morris LL, et al. (2014). A consideração terapêutica visa o alívio sintomático e a modulação do processo autoimune. Duas principais abordagens terapêuticas, com drogas que aumentam o GABA e agentes imunomoduladores são sugeridos com base na patogênese do SPS.

Os benzodiazepínicos são a primeira linha de tratamento, e o Diazepam é prescrito por suas propriedades relaxantes musculares e ansiolíticas como agonista do GABA-A. O Baclofeno é usado juntamente com Diazepam como atividade agonista do GABA-B para controlar a espasticidade Medicamentos GABAérgicos de segunda linha, como Gabapentina (semelhante ao GABA), Vigabatrina (inibidor da GABA-transaminase), Tiagabina (bloqueiam a recaptação do GABA) e Valproato de sódio (aumentam a transmissão do GABA) podem melhorar os sintomas do SPS. O Levetiracetam foi aplicado para tratar com benefício dos sintomas.

Além disso, Tizanidina, um $\alpha 2$ do receptor adrenérgico ( $\alpha 2$-AR) agonista, e toxina botulínica (inibidor da libertação de acetilcolina) foram usadas para tratar a espasticidade com melhora descrita por Pakeerappa PN, et al (2015). Os corticosteróides têm sido frequentemente usados como monoterapia ou em combinação com outros agentes para melhorar espasmos. Outros agentes imunomoduladores, como Micofenolato de Mofetil, Azatioprina, Ciclofosfamida, Ciclosporina, Tacrolimus e Sirolimus também podem fornecer benefícios variáveis. A plasmaférese pode melhorar os sintomas com SPS grave em estudos anteriores. E, finalmente, foi relatado que o rituximabe elimina com sucesso as células $B$ produtoras de autoanticorpos no sistema nervoso central de pacientes com SPS refratário, diminuindo rapidamente o Ab anti-GAD (YI-YIN L, et al., 2019).

Em suma Goran R e Kay FM (2012); descreve que o tratamento SPR baseia-se na sua patogênese, pela qual se indica dois tipos de tratamento: 1) fármacos GABA-agonistas e 2) agentes imunossupressores ou 
imunomoduladores. Sendo a terapia inicial feita com benzodiazepínicos, que aumentam o efeito GABA nos receptores alfagabaérgicos, também se pode utilizar 0 baclofeno, enquanto os tratamentos imunossupressores se reservam aos casos graves. Nesse paciente, houve boa resposta clínica ao uso de benzodiazepínicos, que vai de encontro à primeira linha de tratamento descrita, o uso de imunossupressores não pode ser levado em consideração devido ao quadro de DM1 associado.

A SPR é uma condição neurológica rara, caracterizado por rigidez e espasmos musculares episódicos. Apesar de ser quase impossível esquecer suas características clínicas clássicas, por se tratar de uma entidade rara, a SPR pode ser subdiagnosticada por outras especialidades médicas. Um importante critério diagnóstico é a presença de atividade motora contínua na eletromiografia, a atividade motora contínua pode ser registrada em muitos músculos axiais e também nos músculos da perna e do braço proximal, entretanto, uma eletromiografia normal não afasta o diagnóstico quando os sinais clínicos são exuberantes, e uma prova terapêutica deve ser proposta, como ocorreu no presente caso.

Apesar de se tratar de uma entidade rara, essa hipótese diagnóstica merece ser investigada em pacientes com acometimento muscular com poliendocrinopatias concomitantes, devido à alta prevalência dessa associação e ao grande impacto que essa patologia gera na vida do paciente. Médicos devem estar atentos à possibilidade dessa síndrome para realização de investigação diagnóstica e tratamentos adequados precocemente, tendo em vista que o tratamento pode ser feito com fármacos de fácil acesso e devido ao alto impacto que isso ocasiona na qualidade de vida dos pacientes.

\section{REFERÊNCIAS}

1. APODACA FJC, et al. Síndrome de la Persona Rígida: Reporte de un caso. Rev Med UAS, 2018; 8: 4.

2. BLUM P, JANKOVIC J. Stiff-person syndrome: An autoimmune disease. Mov Disord., 1991; 6: 1220.

3. CARRILHO P, et al. Efficacy of levetiracetam in case of stiff-person syndrome - Case report. Revista Brasileira de Neurologia e Psiquiatria, 2014; 18(1): 68-72.

4. ESPAY AJ, CHEN R. Rigidity and spasms from autoimmune encephalomyelopathies: stiff-person syndrome. Muscle Nerve. 2006; 34: 677-690.

5. GORAN R, KAY FM. Autoimmune stiff person syndrome and related myelopathies: Understanding of electrophysiological and immunological processes. Muscle Nerve., 2012; 45(5): 623-634.

6. GRIMALDI LM, et al. Heterogeneidade de autoanticorpos na síndrome do homem rígido. Ann Neurol., 1993; $34: 57-$ 64.

7. HELFGOTT SM. Stiff-man syndrome: from the bedside to the bench. Arthritis Rheum., 1999; 42(7): $1312-1320$.

8. LINO VC. Síndrome da pessoa rígida: avaliação de 14 pacientes. 2016. $108 \mathrm{f}$. Dissertação (Mestrado) - Escola Paulista de Medicina, Universidade Federal de São Paulo (UNIFESP), São Paulo, 2016.

9. LIRAN O, et al. Stiff Person syndrome: a tough and rigid case. Isr Med Assoc J., 2015; 17(4): $261-262$.

10. MOERSCH FP, WOLTMAN HW. Progressive fluctuating muscular rigidity and spasm (stiff-man syndrome): report of a case and some observations in 13 other cases. Mayo Clin Proc, 1956; 31: 421-427.

11. MORRIS LL, et al. Redução do excesso de rigidez na Síndrome da Pessoa Rígida usando TCC: Um estudo de caso. NeuroRehabilitation., 2014; 35: 627-631.

12. PAKEERAPPA PN, et al. Toxina botulínica, injeção de músculos paraespinhais faciais e cervicais em um paciente com síndrome da pessoa rígida: relato de caso. PM R., 2015; 7: 326-328.

13. RAKOCEVIC G, et al. Anti-glutamic acid decarboxylase antibodies in the serum and cerebrospinal fluid of patients with stiffperson syndrome: correlation with clinical severity. Arch Neurol., 2004; 61: 902-904.

14. SOLIMENA M, et al. Autoantibodies to GABA-ergic neurons and pancreatic beta cells in stiff-man syndrome. $\mathrm{N}$ Engl J Med., 1990; 322: 1555-1560.

15. URBAN GJ, et al. Elevated serum GAD65 and GAD65-GADA immune complexes in Stiff Person Syndrome. Sci Rep., 2015; 5: 11196.

16. YI-YIN, et al. Association of stiff-person syndrome with autoimmune endocrine diseases. World Journal of Clinical Cases., 2019; 9: 2942-2952. 